\title{
O QUE SABEMOS SOBRE O USO DE ESTRATÉGIAS NO ENSINO DE ALEMÃO COMO LÍNGUA ESTRANGEIRA?
}

\section{What do we know about the use of strategies in the teaching of German as a foreign language?}

\author{
Adriana SCHLENKER, Celin-UFPR ${ }^{1}$
}

RESUMO:Este relato tem como foco o uso de estratégias para o estudo e ensino do Alemão como Língua Estrangeira. Aprender uma Segunda Língua ou uma Língua Estrangeira, exige, entre outras atividades, o uso de estratégias que facilitem o aprimoramento do idioma. Considerando que cada aluno tem determinado tipo de estudo, é possível que eles se utilizem das estratégias, que aprimorem seu desempenho nos estudos. Ao conhecer seu estilo e o mecanismo das estratégicas, o aluno poderá desenvolver uma forma mais autônoma e segura no aprendizado da Língua Estrangeira. As considerações aqui relatadas e propostas,-visam não somente repensar o uso das estratégias quanto à aplicação destas em sala de aula, mas também em abrir diálogo entre os professores de alemão.

PALAVRAS-CHAVE: Aprendendo Estratégias; Estratégias no Estudo; Língua Estrangeira, Segunda Língua; Autonomia; Estratégias no Ensino; Alemão.

\begin{abstract}
The current paper focuses on the usage of strategies for the studying and learning of German as a foreign language. Learning a second or foreign language demands, inter alia, the usage of strategies which facilitate the language enhancement. Taking into consideration that each student has a determined sort of studying, they may use strategies which improve their performance when studying. By knowing their style and the strategies mechanisms, the student will be able to develop a more autonomous and safer method to learn a foreign language. The reported and proposed considerations of this paper aim not only at rethinking the use of strategies and their application in the classroom, but also at opening a dialog among German teachers.
\end{abstract}

KEYWORDS: Learning Strategies; Strategies of Studying; Foreign Language;Second Language; Autonomy; Teaching Strategies; German

\footnotetext{
${ }^{1}$ Professora de Língua Alemã no Celin - UFPR. Mestre em Alemão como Língua Estrangeira pela Universidade de Leipzig.
} 
Estratégias de aprendizagem no estudo de Línguas Estrangeiras é um tema de meu interesse há muito tempo, não somente por estudar e lecionar a língua alemã, mas por aprender a desenvolver técnicas que otimizam o aprendizado do idioma. Rebecca Oxford sintetiza que idiomas, estilos e estratégias de aprendizagem ${ }^{2}$ estão entre os principais fatores que ajudam a determinar como e quão bem nossos alunos aprendem uma segunda língua ou uma língua estrangeira (OXFORD, 2003: 1). Aprender uma Segunda Língua é aprender o idioma no local onde este é a comunicação diária entre os demais, onde existe abundante motivação linguística; já aprender uma Língua Estrangeira é aprender um idioma em um meio onde a comunicação não se dá no idioma que se estuda e, assim, a motivação de interação é restrita (OXFORD). O foco deste relato é o estudo e o ensino do Alemão como Língua Estrangeira no CELIN, porém as estratégias e estilos estão relacionados com as duas formas de aprendizagem de um idioma.

A palavra Strategy vem do antigo Greco strategia, que significa as ações tomadas de maneira geral, na intenção de ganhar uma guerra. Esta idéia permanece no mundo atual, porém não mais no sentido de guerra, mas no campo do controle e de atingir objetivos (2003: 362$)^{3}$. A definição do termo Learning Strategies (aprendendo estratégias) é apresentado em diferentes literaturas não somente com termos um pouco divergentes, mas também com conceitos um pouco diferentes entre si. Estes aspectos não serão aprofundados no momento, porém algumas definições serão abordadas, sendo importante salientar que alguns autores partem da definiçao deste termo, tomando como base a definiçao proposta pelo dicionário The New Oxford Dictionary of Englisch, o qual afirma que uma estratégia is a plan of action or policy designed to achieve a major or overall aim (1998: 1837) ${ }^{4}$. O’Malley and Chamot definem este termo com base na teoria cognitiva do IF (se) e THEN (então),

If the goal is to comprehend an oral or written text, and I am unable to identify a word's meaning, THEN I will try to infer the meaning from context (1990: 52)

2 "Language learning styles and strategies." (todas as traduçoes deste relato feitas pela autora)

3 Steps or actions generals take for the purpose of winning a war (in Oxford, 1990).

4 Plano de açao ou método/estratégia designada para atingir o principal objetivo ou global. 
Peter $\mathrm{Gu}$ trabalha a partir da perspectiva de um protótipo que define que a aprendizagem de estratégias não é um conceito que se define claramente, porque as estratégias diferem entre si

Learning Strategy is not a clearly definable concept, because strategies differ from each other in terms of "family resemblance" or "graded degrees of membership (2005: 1).

$\mathrm{O}$ autor Macaro abrange uma série de teorias e nomenclaturas sugeridas por diferentes autores sobre o termo em questão, como o termo skills usado por Harris (1998: 135), o qual gera algumas controvérsias (veja Macaro, Ernesto, 2006: 322-5,). É necessário salientar que o foco sobre o uso de estratégias neste relato, parte principalmente dos aspectos abrangidos por Rebecca Oxford em Language learning styles and strategies (2003), onde há uma visualização sobre os diferentes estilos e estratégias de Learning Strategies, e que também está relacionado com a proposta deste relato sobre o ensino e estudo do Idioma Alemão no Centro de Língua e Interculturalidade - CELIN.

Para se utilizar de estratégias é preciso que o aluno saiba não somente reconhecêlas, mas também utilizá-las, podendo assim escolher as mais compatíveis com seu estilo. Cada aluno tem um estilo diferente de aprender. Segundo Rebecca Oxford, aprendendo estilos Learning Styles é a forma de aproximação generalizada utilizada pelo aluno para com o objeto ou a língua de estudo, por exemplo: de forma global, analítica, auditiva ou visual (...). Os estilos mais significativos dizem respeito a sensory preferences, personality types, desired degree of generality, and biological differences (2003: 360) ${ }^{5}$. Neste ponto segue uma curta descrição sobre a característica destes estilos e em seguida sobre as estratégias. Sensory preferences é a forma física e perceptiva mais confortável de estudo para o aluno, e pode ser dividido em 4 áreas principais: visual, auditivo, cinestésico e tátil.

O estilo visual é a maneira em que os estudantes dão preferência à leitura, à qualquer outra atividade como, seminários, conversação ou mesmo instruções que não tenham como suporte a estimulação visual. O suporte visual para estas atividades é essencial para que este estilo incorpore de forma eficiente outras estratégias. Diferente deste estilo, os estudantes de estilo auditivo aproveitam melhor das atividades orais, como os seminários, e tem maior proveito da comunicação em grupo, bem como de atividades de

5 preferências sensoriais, tipos de personalidade, grau desejado de conhecimento e diferenças biológicas. 
interação, sem a necessidade de estímulos visuais. Estudantes cinestésicos ou táteis dão preferência às atividades de movimentação e gostam de trabalhar com atividades manuais, como colagens e jogos. Ficar sentado por horas não é para estes alunos.

Personality types é outro aspecto importante no ensino da Língua Estrangeira (L2 ou LE), pois abrange diferentes personalidades de uma pessoa como por exemplo: extrovertido ou introvertido. Desired degree of generaliti abrange o contraste entre estudantes que tem o foco em diferentes pontos de um problema. $\mathrm{O}$ foco pode estar na idéia geral ou se concentrar nos detalhes do dado problema, chamado assim de: estilo global e estilo analítico. Biological differences, são as diferenças no ritmo biológico de cada estudante. Por exemplo, alguns alunos tem maior facilidade de estudo pela noite e outros pela manhã. Há também aspectos que envolvem a alimentação, ou não, durante o estudo, e também o local de estudo. Alguns precisam de lugares fechados, sem influência externa, outros precisam de movimento à sua volta.

Segundo esta diferenciação proposta por Oxford, entendo que como professores, somos responsáveis para que os alunos percebam qual seu estilo de estudo, e que assim possam não só compreender o uso das estratégias para que a aprendizagem deles seja mais efetiva, mas também escolher e/ou desenvolver a melhor para seu estilo. Schmeck observou que se uma pessoa tem uma tendência para usar a mesma estratégia em diversas situações we can suspect the presence of a style (1988: 8). Learning Strategies é definido por Scarcella and Oxford, como ações específicas, tal como o comportamento, nível e técnica, utilizada pelos estudantes para melhor desempenho nos seus estudos (1992: 63).

When the learner consciously chooses strategies that fit his or her learning style and the L2 task at hand, these strategies become a useful toolkit for active, conscious, and purposeful selfregulation of learning" (Oxford, 2003: 359).

Oxford classifica o aprendizado de estratégias em 6 tipos: cognitive, metacognitive, memory-related, compensatory, affective and social (2003: 359) ${ }^{6}$. Estas classificações serão descritas de forma concisa, segundo Oxford. A estratégia cognitiva permite a manipulação do material de estudo de maneira direta, através da razão, análise, resumo, reorganização de conteúdo para elaborar diagramas mais avançados (estruturas de

6 cognitivo, metacognitivo, relaçao de memória, compensaçao, afetivo e social. 
conhecimento). Já a estratégia metacognitiva é a administraçao sobre o processo de estudo de maneira global (identificando seu próprio estilo de estudo e suas necessidades, planejamento para uma tarefa de LE, acumulando e organizando materiais, determinando o espaço e o planejamento de estudo, monitorando erros e avaliando o êxito em tarefas, bem como qualquer tipo de estratégias de estudo). A relação de memória permite aos estudantes associações de um ítem ou conceito da LE com outro, mas nao exige necessariamente profundo conhecimento a respeito, por exemplo, o rítmo (grava os sons das palavras ou as combinações entre elas), imagens (uma imagem mental a respeito da palavra ou a idéia sobre o significado da palavra), palavras-chave, entre outras. A estratégia de compensação é conhecida entre muitos através de guessing from context, ou seja, adivinhar pelo contexto, seja ouvindo ou lendo. Outra maneira faz uso de sinônimos e falando alto a palavra em questão, ou mesmo também se utilizando da escrita. Gestos são estritamente utilizados para a atividade de conversação, ajudando o aluno a evoluir seu conhecimento. $\mathrm{O}$ último termo affective and social strategies se ocupa em identificar o nível de humor, receio, preocupação de um estudante, falando sobre sensações, recompensando por um bom desempenho e utilizando de palavras positivas -self talk- para incentivo. Há estudos onde o uso desta estratégia não resultaram de forma positiva, como relata Oxford (2003: 364).

Neste contexto as estratégias podem ser utilizadas de maneira consciente ou inconsciente Blindes Strategietraining ou Informiertes Strategietraining. A questão em explicitar aos alunos, ou não, de que o professor está fazendo uso de uma estratégia, é uma questão pessoal. Porém muitos autores são da opinião de que o aluno deve fazer uso de maneira consciente das estratégias, bem como conhecer seu estilo. Segundo Oxford, também o professor deve tentar assessar o estilo dos aluno e suas estratégias, porque este assesso gera maior entendimento a respeito dos mesmos. O mesmo vale para os professores, os quais deveríam conhecer e assessar seus próspios estilos e estratégias, a fim de estarem cientes das preferências e possíveis preferências de seus alunos (2003: 365). Também é válido salientar que as estratégias e o seu treino e uso, não estão relacionadas como sendo uma orientação ou dica, a qual somente é utilizada no momento em que há um problema a se revolver, no sentido o que se deve fazer, quando se quer estudar algo (Metzig, 2000: 5) ${ }^{7}$, mas sim como uma ferramenta a ser utilizada pelo aluno no processo

7 “...was man machen muss, wenn man etwas lernen möchte.” 
de estudo, ou seja o que se poderia fazer, para criar um processo mais eficiente de estudo e melhorar estes resultados (Desselmann, 1988: 150) ${ }^{8}$, como salienta Chudak Auf neuen Wegen. O uso e ensino de estratégias, é segundo o autor, um trabalho contínuo e sistemático, desenvolvido ao longo do período de estudo, o qual é implementado nas diferentes fases e também objetivamente treinado, revisado e controlado. Os alunos não deveriam ser "ensinados" pelo professor, mas sim o conhecimento e experiência em estratégias do professor, inserido no processo de aprendizagem do aluno, ou seja:

Exigido é a açao integrativa, o que significa, que as técnicas de estudo são adquiridas nas atitudes, ao invés de serem aprendidas em liçoes teóricas (vgl. Rampillon pág 18, 1993, pág 75ff, 1994, pág 124ff, 1996) ${ }^{9}$.

Desta maneira as chances de sucesso, onde o professor tanto treina e usa as estratégias com os alunos Blind Strategie, como também os coloca ao conhecimento de como a construção e os efeitos das estratégias e em quais áreas devem ser usadas Informiertes Training, são ofertadas aos alunos de forma global e compacta no uso de estratégias (Chudak, 2007: 128).

Como aluna de alemão há mais de uma década e, atualmente, professora do idioma no Celin ${ }^{10}$, pude perceber que muitos exercícios são realizados de forma inconsciente, ou seja, os alunos não têm a consciência de que estão se utilizando de uma estratégia, ou que esta seja uma estratégia para que determinado conteúdo seja aprendido Blindes Strategientraining. Um exemplo são os próprios exercícios nos livros didáticos ou atividades relacionadas à memorização, como os "jogos de memória". Na brincadeira em grupo ou em duplas, o aluno memoriza novas palavras e expressões, sem conscientemente perceber a estratégia. Muitos alunos percebem a sistemática de determinado exercício, mas não os ocorre de abordá-las como uma estratégia que pode vir a ser trabalhada e desenvolvida. De outra maneira, a forma consciente na aplicaçao de uma estratégia Informiertes Strategietraining pode vir a assegurar um aprendizado mais efetivo e de longo prazo, como já mencionado anteriormente. Finkbeiner cita que Tönshoff (1995) recorda três elementos utilizados para resolver um problema, ter objetivos claros, intencionalidade

\footnotetext{
$8 “$ “.. was man machen könnte, um den Lernprozess effizienter zu gestalten und seine Ergebnisse zu verbessern."

9 "Gefordert wird der integrative Einsatz, was bedeutet, dass Lerntechniken handeld erworben, anstatt in Theorielektionen gelernt werden müssen."

10 CELIN - Centro de Línguas e Interculturalidade da UFPR.
} 
e consciência de estratégias, como o critério mais importante no aprendizado de estratégias ${ }^{11}$. Um aluno relatou certa vez que ele fazia cartões de papel sulfite, nos quais ele escrevia de um lado a palavra em alemão e do outro a tradução. À medida que ele ia acertando o seu significado, tanto do alemão quanto do português, ele ia retirando estes cartões do grupo e os colocando em outro. Outra estratégia que normalmente é passada aos alunos pelos professores já nos primeiros mêses de estudo da Língua Alemã, é a de atribuição de cores para os três gêneros do idioma. Me recordo de tê-la aprendido ainda bem no início de meus estudos e também de que era uma estratégia de memorização e de ganho de vocabulário. Acredito que muitos professores e alunos se identifiquem com a mesma situação, e pode ser também que ela não tenha sido explicitada como uma estratégia e de como esta está relacionada ao estudo, mas sim como mais um exercício entre tantos outros.

No estudo do idioma aumenta a cada lição o vocabulário, e com isto, aumentam também os substantivos, que precisam de seus respectivos gêneros "der" para masculino, "die" feminino e "das" neutro. A estratégia consiste em designar para cada gênero uma determinada cor: der- azul, die- vermelho e das- preto, ou da cor que o aluno preferir. Sugere-se formar três colunas em um caderno de anotações, e nestas escrever os novos substantivos, que a cada lição são trabalhados, na coluna designada para sua cor-gênero:

\section{der Mann die Dame das Mädchen \\ (o homem) (a mulher) (a menina)}

Para nós, brasileiros, o gênero neutro não existe e muitas palavras em português tem outro gênero em alemão: "a lua - der Mond" e "o nariz - die Nase", e assim esta estratégia é muito bem vinda. Para melhor ilustrá-la, está anexado a este relato um pôster ${ }^{12}$ que a esquematiza e também é apresentada aos alunos do CELIN. A estratégia neste pôster consiste no processo de transformação mental de um substantivo + gênero ainda não memorizados representados pela cor "cinza" em um substantivo na cor referente ao seu

11 Ver: http://webdoc.gwdg.de/edoc/ia/eese/artic98/finkb/10_98.html\#kap03 (último acesso 19.04.2013) " regards the three elements of taking a problem-solving stance, having clear aims/intentionality and (potential) awareness of strategies as the most important criteria for learner strategies".

12 Este pôster foi desenvolvido em grupo pelos alunos durante o Mestrado na Universidade de Leipzig. 
gênero: um gato se transforma em um gato vermelho (die Katze), uma casa, em uma casa preta (das Haus) e um cachorro fica azul (der Hund). Uma maneira mais dinâmica desta mesma estratégia, abrange a afixaçao de bolinhas coloridas pelos objetos da casa e na casa, e junto delas a palavra do objeto. Quando a palavra é memorizada, ainda se mantêm o adesivo colorido para não que não se esqueça de seu gênero. Quando este também está memorizado, retira-se o adesivo (a bolinha colorida), ficando assim o objeto memorizado na cor correspondente ao seu gênero.

O estudo através de cores pode ser relacionado ao estilo cinestésico de estudo, synesthesia is an automatic connection between two ore more senses or sensory modes (Cytowic, 2000). Bachmann expõe em seu artigo, que como aprendiz de francês, ela relacionava as cores aos gêneros dos substantivos. Assim quando precisava recordar o gênero da palavra, bastava lembrar da cor desta palavra. Ela menciona que há suspeitas de que este estilo e a aprendizagem de Linguagem/ Idiomas, podem estar localizados no mesmo hemisfério cerebral, um junto ao outro. Diversas pesquisas apontam uma divisão de nosso cérebro em diferentes áreas e sub-áreas. As mais significativas para a aprendizagem da Língua são o hemisfério esquerdo e o direito. Hinnes afirma que há uma grande diferença na natureza cognitiva dos dois hemisférios, de que o esquerdo domina e suprime o hemisfério direito, e que se o direito é liberado desta supressão, há um resultado mais efetivo no processo de aprendizagem, e também uma diferenciação entre estes dois lados, como por exemlo, de que o lado esquerdo está relacionado à "consciência, ao indutivo, lógico, linear e questina o porquê e o como", já a característica do lado direito é o "subconsciente ou mesmo inconsciente, dedutivo, intuitivo, não lógico e nem linear". Acrescenta que já na década de 60 e 70 as pesquisas afirmarvam que o hemisfério esquerdo está ligado ao processo verbal, ou seja, ele processa e estimula o processo linguístico, sendo que o direito está ligado ao processo não verbal, e que não estimula o processo linguístico (1987: 600-1) $)^{13}$ ".

Dentro deste âmbito, me pergunto o que seria mais efetivo em termos de estratégia para aprendizagem do idioma alemão: se o aluno primeiro deve desenvolver o raciocínio para resolver as atividades propostas e depois tomar conhecimento da estratégia, ou o contrário, saber como funciona a estratégia, conhecer sua designação e aí colocá-la em uso? A meu ver, para um aluno poder, de forma consciente e autônoma, optar por uma ou

13 http://www.jstor.org (último acesso 21.04.13) 
outra estratégia, ou mesmo utilizá-las, ele precisa primeiramente conhecer seu estilo e conhecer também o mecanismo que facilita o estudo, ou seja, a estratégia. Mesmo achando efetivo o trabalho com os exercícios em que a estratégia não é explícita, como professora tenho a tendência de priorizar a explicitação de diferentes estratégias para os estudos. Levando em conta os diferentes estilos, é válido dizer que a estratégia que para um aluno é um diferencial substancial em seus estudos, pode não ser para outro, ou melhor dizendo: uma estratégia não é boa nem ruim; é neutra (...)(OXFORD, 2001: 362) ${ }^{14}$. Peter Yongqi Gu cita que O'Neil (...) define que aprender estratégias é uma aptidão intelectual e afetiva que constitui a condição necessária para um estudo mais eficiente (1978: p. xi) ${ }^{15}$.

Ainda mantendo o foco nas estratégias, gostaria de mencionar o resultado positivo ao se trabalhar com os alunos em sala de aula com atividades que exigem movimentação e promovem a interação entre o grupo. Esta interação através do movimento, também pode ser relacionada ao estilo cinestésico de aprendizado, como mencionado anteriormente. Breithecker afirma que atividades corporais tem uma influência incentivadora sobre a atenção e a capacidade de concentração. Através de atividades motoras a estática corporal e mental são superadas (pág.5) ${ }^{16}$. Gostaria de mencionar o trabalho estudo para o desenvolvimento de movimento, jogos e esporte em escolas integrais, que oferece uma visualização sistemática de exemplos, critérios, materiais, avaliação, e autores nesta área, focado aos profissionais do ensino ${ }^{17}$. Autores como Brauneck 1993, Grädel 1996, Bräuer 1995, Barba 1993, abordam as questões não só de movimento em sala de aula, mas atreladas ao ganho de conhecimento e interação durante as aulas. Jogos didáticos, brincar aprendendo a Língua, jogos criativos, movimentação entra em jogo e jogos interativos, são alguns termos utilizados por Hallet para obter um ganho qualitativo no estudo (2002). Outro termo utilizado nesta área é o estudo cooperativo Kooperatives Lernen: O estudo cooperativo acontece, quando dois ou mais estudantes tendo o mesmo nível de

14 "A given strategy is neither good nor bad; it is neutral."

15 "defined learning strategies as intellectual and affective skills that constitute a necessary condition for more efficient learning".

16 "körperliche Tätigkeiten haben einen förderlichen Einfluss auf Aufmerksamkeit und

Konzentrationsfähigkeit. Durch motorische Aktivität wird körperliche und geistige Statik

überwunden".http://www.haltungbewegung.de/Data/Sites/4/media/dokumente/schule/projekte/BAG4_D. pdf (último acesso 21.04.13)

17 http://www.uni-marburg.de/fb21/ifsm/ganztagsschule/schulmaterial/bewegung-im-unterricht-literatur (último acesso 12.04.13) 
conhecimento se ajudam mutuamente para resolver uma atividade, um exercício para atingir um objetivo em comum (Hinde/Groebel, 1989 in Benkmann, 1997: 88) ${ }^{18}$.

Independente da forma utilizada, estas atividades desenvolvem uma dinâmica que impulsiona a aprendizagem em sala de aula, como afirma Wolff na Tese 9:

(...) estudar é um processo que os alunos controlam através das estratégias $(\ldots)$ e que estudar é um processo, onde o trabalho em grupos resulta em sucesso (1997: 48). ${ }^{19}$

Pessoalmente, noto a dinâmica e o despertar dos alunos em sala de aula, quando atividades de movimentação e interaçao são utilizadas, impulsionando assim ao aprendizado. Por este motivo, sou a favor de que o professor de idiomas conheça algumas estratégias para os diferentes estilos de aprendizagem e exponha estas, à sua maneira, aos alunos. Também acredito que, principalmente na atualidade, os estudantes de Língua Estrangeira tenham uma dinâmica muito maior do que há alguns anos atrás, e que não se motivem ao ter uma aula frontal onde o monólogo explicativo não ceda espaço para a interação. $\mathrm{O}$ desinteresse dos alunos pelo conteúdo aumenta, ao meu ver, gradativamente. Um fator relevante pode se relacionar às novas mídias ligadas à Internet. Esta tem grande atuação na atual dinâmica interativa de nossos alunos. Já nas escolas, por exemplo, são utilizados através da conexao da Internet, exercícios Online para o estudo, e praticamente cada aluno tem seu telefone celular com conexão, ou seja os alunos já vão à escola com bastante motivação e conhecimento de uso destas ferramentas. O uso da Internet também é uma ferramenta estratégica da atualidade

Muitos professores e também alunos descobriram a Internet e são fascinados pela vasta possibilidade de pesquisa, da comunicação por E-Mail e Chat, de acesso à informações e materiais atuais (Freibichler, 2000: 7) ${ }^{20}$.

18 „Kooperatives Lernen findet statt, wenn zwei oder mehr relativ gleichberechtigte Partner sich wechselseitig dabei unterstütuen, eine gemeinsame Aufgabe zu bewältigen, ein gemeinsames Ziel zu erreichen."

19 "Lernen ist ein Prozess, der von den Lernenden durch Strategien gesteuert wird; und Lernen ist ein Prozess, der in Gruppen besonders erfolgreich abläuft; (...)."

20 "Viele Lehrer und auch Lernende haben das Internet entdeckt und sind fasziniert von den Möglichkeiten der Informationsrecherche, der Kommunikation über E-Mail und Chat, vom Zugriff auf aktuelle Informationen und Materialien." 
Como o uso da Internet e os exercícios Online não são o tema proposto no momento, estes não serão abordados além do mencionado. Mantendo o foco sobre a movimentação e as estratégias, Wolff define em sua tese 5:

É pressuposto, ... que eles no processo de aprendizado experimentam mudanças, que dependem do seu conhecimento já adquirido, de suas estratégias de estudo e de seu estilo (1997:48). ${ }^{21}$

Neste contexto, fica a pergunta se os professores de Línguas Estrangeiras estão a favor, abertos, para que seus alunos reconheçam por eles mesmos e controlem as estratégias. Quais tipos de estratégias os professores utilizam em sala de aula e quais destas são expostas aos alunos como tal? Poderiam os professores também ordenar estas estratégias com os diferentes estilos de aprendizagem? Uma pesquisa entre os docentes poderia definir não somente as estratégias e seu uso no ensino de Línguas Estrangeiras, nesse caso o idioma alemão, mas também traçar um perfil dos nossos alunos, primeiramente no CELIN, podendo se estender a uma pesquisa envolvendo outras escolas de idiomas.

Tais estratégias já são utilizadas em vários campos de nossas vidas, seja para marcar datas de aniversários, senhas, endereços, entre outros. Sua finalidade é facilitar o acesso às informações mentais e, dessa maneira, utilizá-las para o estudo da Língua Estrangeira, sendo esta uma estratégia em desenvolvimento e de suma importância para proporcionar uma aprendizagem de qualidade.

\section{REFERÊNCIAS}

ALEXANDRE, A., GRAHAM, S., \& HARRIS, K.R. A perspective on strategy research: Progress and porspects. Educational Psychology Review, 10, 129-154.

BACHMANN, Michele. Synesthesia and the Language Classroom - Using Personalised Colour Schemes for Language Learning. Humanising Language Teaching, Year 12; Issue 3; June 2010.

CHLOSTA, C., LEDER, G., KRISCHE, B. (Hg). Auf neuen Wegen: Deutsch als Fremdsprache in Forschung und Praxis. 35. Jahrestagung des Fachverbands Deutsch als Fremdsprachen an der Freien Universität Berlin 2007. Fadaf: Band 79.

21 „Es wird angenommen, (...) dass sie beim Lernprozess Veränderung erfahren, die vom Vorwissen des Lernenden, von seinen Lernstrategien und seinem Lernstil abhängen.” 
CYTOWIC, R.E. Synesthesia. A Union of the Senses, $2^{\text {nd }}$ edition. London: MIT Press, 2000.

DESSELMAN, Günther. Lernstrategien und Lerntechniken im Deutsch als Fremdsprachenunterricht. In Deutsch als Fremdsprache 3, 148-155.

FINKBEINER, Claudia. The promotion of explicit and implicit learning strategies in English instruction: a necessary aim? EESE 10/1998, Kassel.

FREIBICHLER, Hans. Multimedia und Internet-Werkzeug zum Sprachenlernen. Erschienen, IN: Tschirner E. u.a. Schnittstellen: Lehrwerke zwischen alten und neuen Medien. Berlin: Cornelsen: 2000.

GU, Peter Yongqi. Learning strategies: prototypical core and dimensions of variation. Editora: [Auckland, N.Z.]: AIS St Helens. Centre for Research in International Education, 2005.

HALLET, Wolfgang: Fremdsprachenunterricht als Spiel der Texte und Kulturen: Intertextualität als Paradigma einer kulturwissenschaftlichen Didaktik. Trier: WVT, 2002.

HINNES, Terence. Left Brain/ Right Brain Mythology an Implications for Management and Training. Academy of Management Review, 1987, Vol. 12, No.4, 600606.

MACARO, Ernesto. Strategies for Language Learning and for Language Use: Revising the Theoretical Framework. The Modern Language Journal, 90,iii, 2006.

METZIG, Werner, Schuster, Martin. Lernen zu lernen: Lernstrategien wirkungsvoll einsetzen. Berlin: Springer. 2000.

O’MALLEY, J.M. \& CHAMOT, A. U. Learning strategies in second language acquisition. Cambridge: Cambridge University Press, 1990.

O'NEIL, H. F., Jr. Learning strategies. New York: Academic Press, 1978.

OXFORD, R. L. Language Learning Strategies: What every teacher should know. Boston: Heinle \& Heinle, 1990.

OXFORD, Rebecca. Language learning styles and strategies. Berlin, Walter de Gruyter, 2003.

TÖNSHOFF, Wolfgang: Lernerstrategien. IN: Bausch, et. al. (eds.) Handbuch Fremdsprachenunterricht. Tübingen/Basel, 1995. 
SCARCELLA, Robin c. and OXFORD, Rebecca 1 . The Tapestry of Language Learning: The Individual in the Communicative Classroom. Boston, MA: Heinle \& Heinle Publishers 1992.

SCHMECK, R.R. Learning strategies and learning styles. London: Plenum Press, 1988. The new Oxford Dictionary of Englisch. Oxford: Oxford University Press, 1998.

WOLFF, Dieter. Instruktivismus vs. Konstruktivismus. Zwanzig Thesen zur Lernbarkeit und Lehrbarkeit von Sprache. München, 1997. 
Artikelprobleme?

象

ถูก

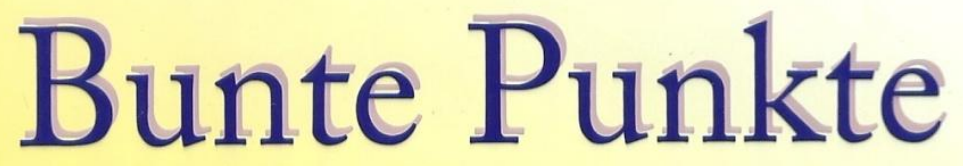
ist die Lösung!!!

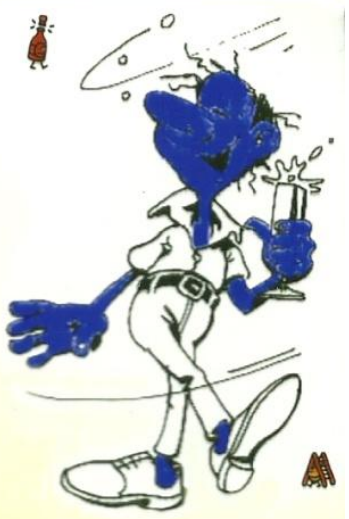

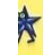
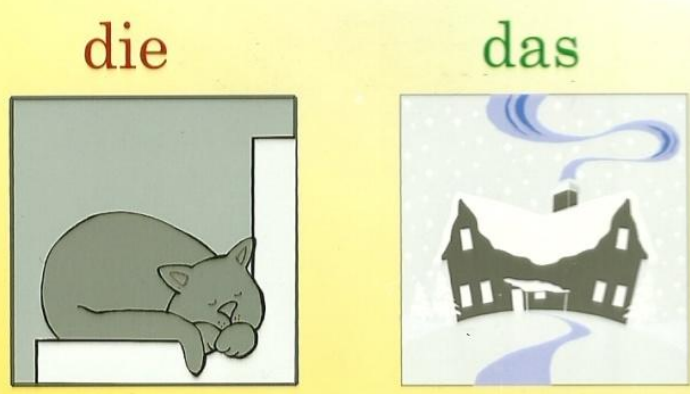

Klebezettel mit Wort und buntem Punkt.
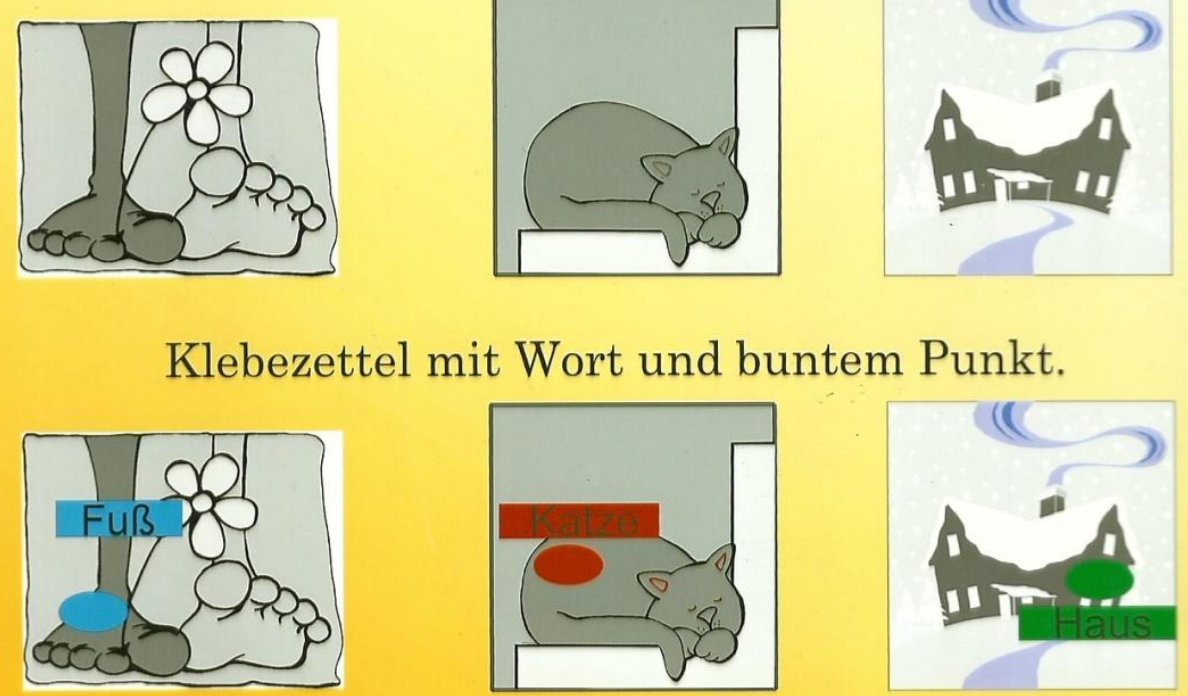

Gelerntes Wort geht weg. Bunte Punkte bleiben.
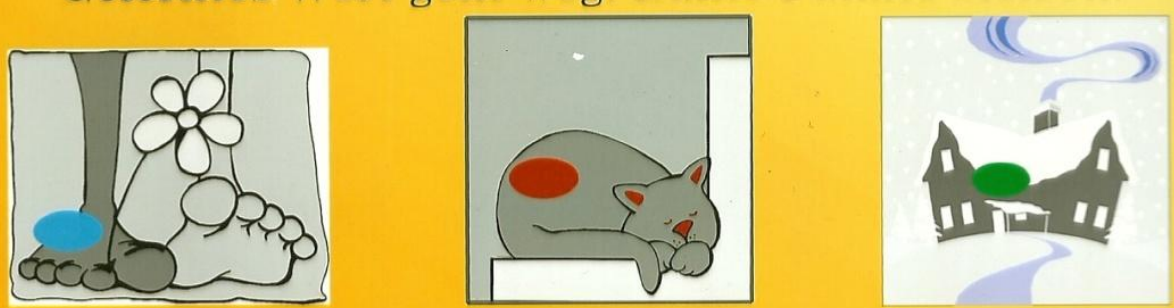

Im Kopf bleibt Gegestand mit Farbe verbunden.
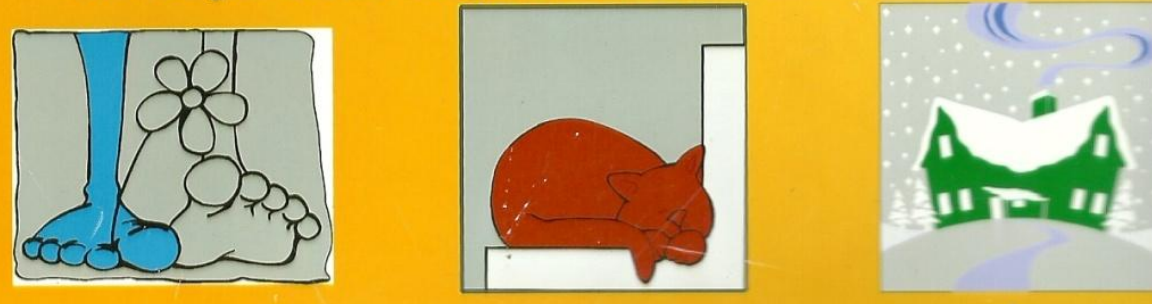